\title{
The Construction of Management Domain Ontology
}

\author{
Dexun Li
}

\author{
Fuyang Teachers College, Fuyang, China 236037 \\ leeadesir@163.com
}

Keywords: Retrieval, Ontology, Domain, Management.

\begin{abstract}
Based on the comparison between management thesaurus and management domain ontology, this article proves the necessities and practicalities of ontology-based management knowledge organization, and the significance of ontological approaches, which should be adopted to management information organization. In addition, it also summarizes the basic situations of management domain ontology, and designs the principles and procedures of management domain ontology establishing, based on which a management domain ontology is established.
\end{abstract}

\section{Introduction}

With the gradual popularization of internet, copy and free expression of digital, electronic, or network-based information resources and its digital text has become possible, which making management information resources augment daily and the informational degree of music increase quickly. Because of the contradiction between rapid growth and effective use of management information, how to provide high-rank management information services, control and develop management information resources effectively, and let it meet the management information needs, has become an urgency.

The knowledge organization based on ontology provide a new space for management information organizations. It can give the management information resources more accurate and complete semantics, and make computer a better understanding of information resources, as a result, the semantical and intelligent of information services will come true.

Based on other domain ontologies which have already been built [1-5], this article attempts to define the nature of management domain ontology from the perspective of servicing management practices, and discusses the principles, procedures and methods of establishing it, finally builds a domain ontology which has management characteristics.

\section{Management Thesaurus and Management Domain Ontology}

Ever since there is storage and processing activities to produce management information, the management information retrieval has become a problem. In order to resolve the contradictions between information retrieval and human information needs, management thesaurus have been using. Though it has simple structure, in fact it is the most primitive ontology.

Management professional thesaurus provides an expression of term structure, but its aim is only involved in the relationships between terms in particular natural language, unhelpful for the definition of concepts, and disregards the expression and meanings of concepts is standard or not. The relationship between concepts has not been shown either. Facing to the needs of various users in the internet environment, it usually can not provide effective management information accurately. Its problems can be outlined as the reliability of expression, the expression diversity and the expression island.

Management Domain Ontology should proceed from the perspective of knowledge, provide the approaches of organizing information resources, and progress towards the automated, integrated and intelligent knowledge organization in the internet environment, and the aim is to find ideal management information exactly. The key lies in improving management information retrieval from traditional keyword-based level to knowledge-based or concepts-based level. The central role of 
ontology is organizing information resources in the perspective of semantics. It defines a particular area, a series of concepts among areas and relationships between concepts, and provides a knowledge expressing language, which is more detailed, standardized, and can express the semantics more comprehensively. With abstract concepts and terminology of ontology, the knowledge structure of management domain can be analyzed more clearly [6].

It is generally believed that ontology is derived from philosophy domain, and used to be a sub-domain of philosophy in a very long period. So far, it covers many fields, such as philosophy, artificial intelligence, knowledge engineering, and so on [7-9]. Management domain ontology includes various of standard terms in management domain, the precise definition to these terms, and the identification of relationships between these terms [10]. It have lots of advantages in knowledge expressing, sharing, reusing, etc. As for management information resources, ontology can provide an intelligent retrieval mechanism, overcome the drawbacks of traditional information retrieval techniques, enhance the processing capabilities of retrieval systems, and really meet users' information needs and habits of expression. It improves management information retrieval from traditional keyword-based level to knowledge-based or concepts-based level, and reduces the terminal retrieval users' cognitive burden at the same time. By this way, it can perfect it keyword-based retrieval, greatly improve the efficiency of management information retrieval, and achieve the intelligent information retrieval ultimately.

\section{Establishing Principles and Procedures}

During management domain ontology establishing, some concepts must be considered, which belong to management, and unique to management, the properties of concepts, the relations between concepts and properties, and so on. The establishment principles and procedures of management domain ontology will be interpreted respectively as following. The management domain ontology establishing should act on the principles such as the standardization of terms, the consistency of concepts definition, the isometric of same semantic level, the economy and the integrity and extensibility.

The establishing of management domain ontology is in a certain order, and follows the procedures of scoping, analysis, associate, accurate expression, inspection, evaluation and maintenance, etc.

Intelligent information retrieval of management domain ontology can represent information of term's meanings. For example, the term marketing is no longer processed as a string, that is to say, there is no longer just a calculation of the frequency of string in a literature, the retrieval tools can develop the terminology relationship and obtain information about marketing directly. When the term marketing is input, system will transfer it to the nodes of concepts, just like the diagram shows above. All the information directly related to this concept can be accessed directly through this relations among terms.

The hierarchical structure is just a frame for the system of concepts, which can not reflect the varied relations between concepts. Therefore, the properties of classes need to be defined. Define slots are such a descriptors used for describing the properties of classes and instances. It can give the formalization description to the natures of concepts, properties and relationships. Here is an example taken from management domain ontology.

Eg.Planning Function

Class Name: \{Planning function \}

Inherit properties: \{all attributes of its upper concept 'management function'\}

Adjacent concepts: \{organizing function, leadership functions, control function, etc. \}

Sub-classes: \{forecast (analysis of environment), decision-making, and plan, etc. \}

Agents: \{managers, including managers of all strata and all fields, etc. \}

Roles: \{act as the entrepreneur, leader, information master, resource distributor, etc. \}

Approaches: \{administrative, legal, economic and other management approaches\}

Functions: \{planning and arrangements for action to be taken in the future beforehand

Objects: \{involving human, financial and material resources, time, space, information etc. \} 
If the ontology we are developing is for macro-management, this ontology can be extended to other management areas, such as public administration, business administration, management science and engineering, agriculture and forestry management, library management, information and records management, and so on. Its class hierarchy model can be diagrammed as in fig.1, which has been put below the references.

In addition, the similar or related concepts can be speculated by use of these relationships. With this approach, users can obtain a particular concept and all information related to it. Users only need to use natural language search query, such as list all marketing information, the most direct answer can be generated automatically by the system, including upper concepts, adjacent concepts and sub-concepts of marketing.

\section{Conclusion}

On the basis of combining ontological approaches with management information resources, this article tries to solve retrieval problems of some users who are interested in and in need of management information resources, and establishes a management concepts structure model by defining the core class, class hierarchy, and the slots. Through semantic-level natural language processing of web document information and users' query, the model has built a knowledge base of management domain, matched the real user query needs with management information resources and its mapping source document, and presented the retrieval results to users after sort processing.

The definite expressing of data semantics and the application of domain theory will push management information services to a higher level, provide users with a high-quality new services, lay foundation and provide instructive precedent and help for the realization of intelligent management information retrieval and the accomplishment of the semantic Web establishment.

\section{Acknowledgements}

This work has been supported by the CEEUSRO Project between the Anhui Kejian Education Investment and Development Co. , Ltd. and Fuyang Teachers College, named A Study on the Incentive and Restraint Mechanism in the Human Resource Management of Private Educational Institutions, to which I am grateful. Early versions of the ideas discussed here were presented at the 13th Chinese Lexical Semantics Workshop (CLSW2012), I am indebted to the the participants of the talk for useful comments and discussions in the writing of this paper. Needless to say, all errors are my own.

\section{References}

[1] Borst W N. Construction of Engineering Ontologies for Knowledge Sharing and Reuse. Doctoral dissertation. University of Twente, Enschede (1997)

[2] Xin Qi, Qianxing Xiong, Yuqiang Li. An e-Learning System based on Domain Ontology. Proceedings of 2007 International Symposium on Distributed Computing and Applications to Business, Engineering and Science(Volume II) (2007)

[3] Xiankun Zhang. Logistics Domain Ontology Model and Its Application. Progress in Measurement and Testing-Proceedings of 2010 International Conference on Advanced Measurement and Test. IEEE CPS (2010)

[4] Ziyu Liu. Research on Construction and Semantic Retrieval of Multiple Majors Domain Ontology. Doctoral dissertation, Beijing Jiaotong University (2010)

[5] Xun Jiang, Yijie Bian, and Mingfeng Wu. Research on Domain Ontology Based Knowledge Retrieval Model. Library and Information Service, No 18: (2010), p.116-119+144

[6] Uschold, M. and Gruninger, M. Ontologies: Principles, Methods and Applications. Knowledge Engineering Review, No 11/2 (1996), p. 93-136

[7] Studer R, Benjamins V R, FenselD. Knowledge engineering, principles and methods. Data and Knowledge Engineering, No 25( 122 ): (1998) , p.161- 197 
[8] Austin T Williams, Ontologies. IEEE Intelligent Systems, No 1/2: (1999), p.18 - 19

[9] Guozheng Xiao, Donghong Ji, and Shan Xiao. The Types of Ontology and the Ontology Structure of Chinese Semantic Web. Yangtze River Academic,No 2: (2011), p.111-117

[10] Chun Chang. Construction and Conversion of Ontology in Agricultural Information Management. Doctoral dissertation, Chinese Academy of Agricultural Sciences, Peking (2004)

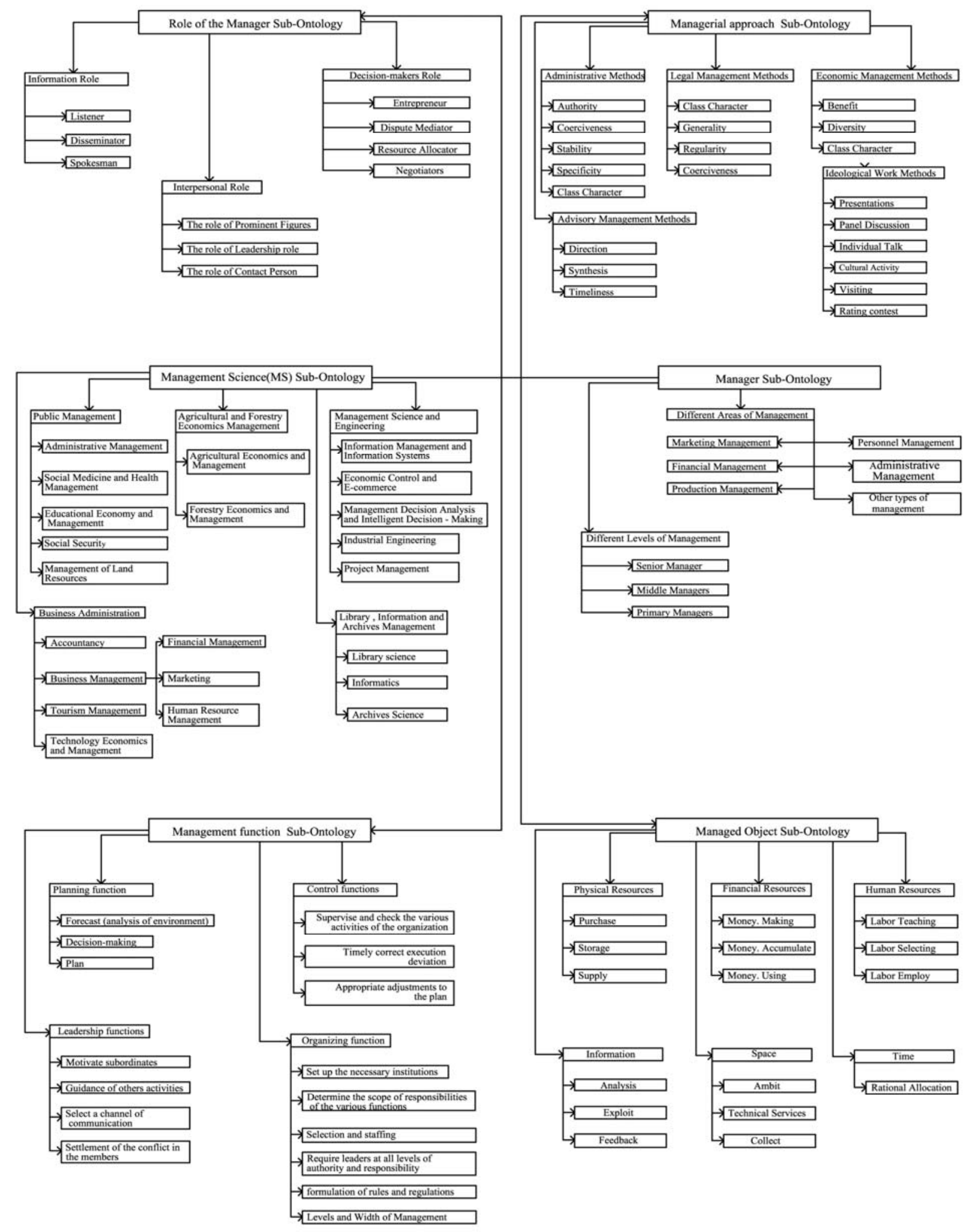

Fig.1. The Management Domain Ontology 